\title{
GRAU DE DEPENDÊNCIA DO PACIENTE EM RELAÇÃO À ENFERMAGEM: ANÁLISE DE PRONTUÁRIOS
}

\author{
Luciana Mahnis Pereira Carmona ${ }^{2}$ \\ Yolanda Dora Martinez Évora ${ }^{3}$
}

Carmona LMP, Évora YDM. Grau de dependência do paciente em relação à enfermagem: análise de prontuários. Rev Latinoam Enfermagem 2003 julho-agosto; 11(4):468-73.

Pretendemos, com este estudo, identificar, na evolução de enfermagem, o grau de dependência do paciente anotado pelo enfermeiro e confrontar com um instrumento de classificação. Fizeram parte do estudo 74 pacientes, 15 enfermeiros e 04 docentes, no período de 22 de setembro a 27 de outubro de 2000. Observamos nos resultados que houve discretas alterações do grau de dependência do paciente, da admissão até a alta, e que os pacientes intermediários apareceram com maior incidência (41,9\%). Porém, para a nossa realidade, ainda devemos treinar os enfermeiros e formular um instrumento próprio de acordo com a característica das unidades de internação.

DESCRITORES: enfermagem; pesquisa em administração de enfermagem; determinação das necessidades de cuidados de saúde

\section{DEGREE OF PATIENT DEPENDENCY IN RELATION TO NURSING STAFF:} ANALYSIS OF CLINICAL RECORDS

In this study, we intend to identify in the evolution of nursing the intensity of patient dependency as it is written down by the nurse and confront it with a ranking instrument. 74 patients, 11 nurses and 04 lecturers were part of the study held from September 22 until October 27, 2000. We noticed slight modifications in the degree of patient dependency from hospital admission to discharge. Furthermore, the majority of the patients that needed help were the intermediate ones (41.9\%). However, our reality still requires nursing training and the creation of an appropriate classification instrument, in accordance with the features of our internment units.

DESCRIPTORS: nursing; nursing administration research; health care needs assessment

\section{GRADO DE DEPENDENCIA DEL PACIENTE CON RELACIÓN A LAS(OS) ENFERMERAS(OS): ANÁLISIS DE HISTORIAS CLÍNICAS}

Pretendimos con este estudio identificar en la evolución de enfermería el grado de dependencia del paciente anotado por el enfermero y confrontar con un instrumento de clasificación. Hicieron parte del estudio 74 pacientes, 15 enfermeros y 4 docentes durante el periodo de 22 de septiembre a 27 de octubre de 2000. Verificamos que hubo alteraciones discretas del grado de dependencia del paciente desde la admisión hasta el alta médica y que hubo una incidencia más alta de los pacientes intermedios (41.9\%). Sin embargo para nuestra realidad todavía es necesario adiestrar los enfermeros y formular un instrumento propio de acuerdo con la característica de las unidades de hospitalización.

DESCRIPTORES: enfermería; investigación en administración de enfermería; evaluación de necesidades

\footnotetext{
${ }^{1}$ Trabalho extraído da dissertação de mestrado apresentada à Escola de Enfermagem de Ribeirão Preto, da Universidade de São Paulo, em dezembro de 2001; ${ }^{2}$ Enfermeira, Mestre em Enfermagem, Professor Assistente da Universidade de Brasília, e-mail: Iumah9704_@hotmail.com.br; ${ }^{3}$ Orientadora, Enfermeira, Professor Livre-Docente da Escola de Enfermagem de Ribeirão Preto, da Universidade de São Paulo, Centro Colaborador da OMS para o desenvolvimento da pesquisa em enfermagem
} 
INTRODUÇÃO

O grau de dependência ou a classificação do paciente foi objeto de estudo de vários autores. No entanto, podemos observar que, na prática, o paciente não é classificado como deveria ser.

Observando as prescrições de enfermagem dos enfermeiros das unidades de internação do Hospital de Clínicas de Marília-SP, notamos que se apresentavam incompletas, pois não consideravam, na prescrição, o nível de dependência do paciente.

Sabemos que, é por meio de uma correta avaliação do paciente, seu estado físico/emocional e grau de dependência da enfermagem, que podemos sustentar um excelente planejamento da assistência.

A liderança no estudo de classificação de pacientes (grau de dependência - Sistema de Classificação de Pacientes "SCP"), indubitavelmente, cabe a um grupo de pesquisadores formado no John Hopkins University and Hospital, nos Estados Unidos, em maio de $1961^{(1)}$.

Durante $024^{\circ}$ Congresso Brasileiro de Enfermagem, em 1972, evidenciou-se a primeira tentativa de dimensionar a dependência de enfermagem no Brasil. Ela descreve o histórico de enfermagem e refere que são identificados os problemas de enfermagem, sendo que estes levam à identificação das necessidades básicas afetadas e do grau de dependência do cliente em relação à enfermagem para o seu atendimento ${ }^{(2)}$.

O grau de dependência pode variar entre parcial ou total. Na dependência total, está implícita a extensão, compreendendo tudo aquilo que a enfermagem faz pelo ser humano, quando este não tem condições de fazer, seja qual for sua causa. Na dependência parcial, a assistência de enfermagem pode situar-se em termos de ajuda, orientação, supervisão e encaminhamento, havendo uma ordenação seqüencial e inter-relacionada dessa assistência, isto é, quando a dependência é de ajuda, esta implica, necessariamente, orientação, supervisão e o encaminhamento, quando couber ${ }^{(2)}$.

A dependência parcial foi expressa, quantitativamente, em graus crescentes, de acordo com a participação do paciente em seu cuidado, sendo os graus 1,2,3,4 e 5. Em 1974, diminuíram essas subdivisões para apenas 4. A determinação dos valores resultava da experiência de cada enfermeira (era empírica). Esses valores eram atribuídos conforme as necessidades básicas de cada indivíduo: conhecimento acerca de sua doença, deambulação, motilidade, estado mental, condições do ambiente e condições socioeconômicas ${ }^{(2)}$.

OSCP permite o planejamento das necessidades de grupos de pacientes; maior satisfação para a clientela, equipe de enfermagem e médicos; maior rendimento e eficiência no trabalho e menor tempo de permanência do paciente no hospital. A adoção do conceito implica inúmeras decisões administrativas quanto à organização, propósitos, planta física e equipamentos, normas operacionais, seleção e treinamento de pessoal, além de critérios e normas para classificação sistemática dos pacientes $^{(3)}$.

Diante desses conhecimentos, surgiu a idéia de procurarmos meios para identificar a melhor forma, na prática, de avaliar o grau de dependência do paciente.

Para tanto, fizemos um levantamento bibliográfico, e deparamos com diversos trabalhos. Um destes nos chamou a atenção( ${ }^{(4)}$, por ser um trabalho recomendado pelo Conselho Federal de Enfermagem ${ }^{(5)}$.

Esses autores ${ }^{(4)}$ implementaram o Cuidado Progressivo ao Paciente (CPP), na Clínica Médica do Hospital Universitário da USP - São Paulo, realocando os pacientes conforme sua classificação por complexidade assistencial, por meio de cinco categorias: autocuidado, intermediário, alta dependência, semi-intensivo e intensivo.

Outros autores também tentaram classificar os pacientes em grau de dependência ${ }^{(6-12)}$.

Isso nos levou a reflexões acerca da Sistematização da Assistência de Enfermagem (SAE), principalmente após as exigências feitas pelo Conselho Regional de Enfermagem de São Paulo (COREN-SP), conforme a Decisão COREN-SP/DIR/008/99 ${ }^{(13)}$.

Não podemos negar que a SAE traz benefícios aos pacientes e, após a exigência do COREN-SP, o Serviço de Enfermagem do Hospital de Clínicas de Marília vem tentando aprimorar esse processo.

Portanto, após essas reflexões e diante dessas necessidades, procuramos, neste estudo, avaliar a demanda do perfil de dependência dos pacientes em nossa prática e também identificar como o enfermeiro tem classificado o grau de dependência do paciente, em sua prática profissional.

\section{OBJETIVOS}

- Identificar, na evolução de enfermagem, o grau de dependência do paciente, anotado pelo enfermeiro. 
- Verificar como os enfermeiros classificam os pacientes em grau de dependência dos cuidados de enfermagem.

- Confrontar a classificação realizada pelos enfermeiros com o instrumento de classificação ${ }^{(4)}$.

- Avaliar a demanda do perfil de dependência dos pacientes da amostra.

\section{MATERIAL E MÉTODOS}

Trata-se de um estudo exploratório-descritivo, realizado em 04 unidades de internação do Hospital de Clínicas de Marília - Unidade I - Alas B1, C, D1A e D1B.

A população de referência foi constituída pelos enfermeiros assistenciais, enfermeiros docenteassistenciais e pacientes das unidades citadas.

Para avaliação dos dados, adotamos alguns critérios de inclusão dos pacientes na amostra:

- permanência hospitalar maior ou igual a quatro dias, para avaliação da demanda do perfil de classificação durante a internação;

- anotação do grau de dependência pelo enfermeiro, no primeiro dia de internação, no meio da internação e no último dia (alta).

Assim, tivemos que excluir 26 pacientes que não apresentavam as características necessárias para análise e comparação dos dados. Fizeram parte do estudo 74 pacientes.

Nos prontuários dos pacientes, foram investigadas as anotações do grau de dependência realizadas pelo enfermeiro. Os enfermeiros foram questionados sobre os recursos que utilizaram para chegar à conclusão da dependência, por meio de um instrumento de coleta de dados, com perguntas semi-estruturadas.

No período de setembro a outubro de 2000 , a pesquisadora consultou os prontuários dos pacientes, realizou avaliação clínica junto ao paciente (história e exame físico), classificou-os conforme instrumento de classificação $0^{(4)} \mathrm{e}$, concomitantemente, verificou a definição de cada área de cuidado para confirmação da classificação. O uso desse instrumento foi autorizado, por escrito, pela autora.

O projeto de pesquisa foi aprovado pelo Comitê de Ética e Pesquisa da referida instituição, e os profissionais enfermeiros e pacientes assinaram o termo de consentimento informado, conforme a Resolução 196/ 96 do Conselho Nacional de Saúde ${ }^{(14)}$.

\section{RESULTADOS E DISCUSSÕES}

A amostra ficou constituída por 74 pacientes, sendo 21 pacientes da Ala B1, 17 da Ala C, 13 da Ala D1A e 23 da D1B; sendo 60,8\% do sexo masculino, e $39,2 \%$, do sexo feminino.

A média de idade constatada foi de 56,3 anos, sendo $39,2 \%$ maiores de 61 anos, e 25,7\%, entre 51-60 anos.

Comparando as idades desses pacientes com as idades dos que internaram no período de dezembro de 1999 a maio de 2000, no referido hospital, houve aumento de pacientes de faixa etária entre 41-60 anos, (14,8\% de diferença), portanto uma clientela cada vez mais idosa.

As doenças que mais incidiram foram as do aparelho circulatório (21,6\%), aparelho digestivo (10,8\%), doenças infecciosas e parasitárias $(9,0 \%)$ e do aparelho respiratório (8,6\%). Com uma média de 3,75 diagnósticos/ paciente.

A média de permanência hospitalar girou em torno de 8,2 dias.

Analisando o grau de dependência do paciente conforme sua classificação por meio do instrumento de classificação $^{(4)}$

O grau de dependência do paciente foi avaliado conforme o instrumento de classificação ${ }^{(1)}$, diariamente. Cabe mencionar que o grau de dependência foi analisado durante a admissão, no meio da internação e na alta do paciente, avaliando a demanda do perfil do paciente durante sua internação.

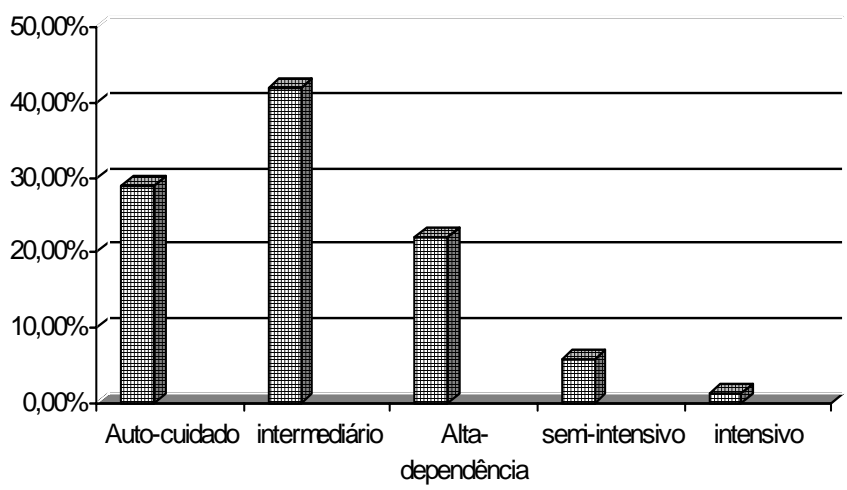

Figura 1 - Classificação dos pacientes internados nas Alas B1, C, D1A e D1B do Hospital de Clínicas de Marília - Unidade I, durante o período de 22 de setembro a 27 de outubro de 2000 , realizada por meio do instrumento de classificação 
Por meio do instrumento de classificação ${ }^{(4)}$, pudemos observar que $28,9 \%$ dos pacientes pertenciam à classificação de autocuidado, 41,9\%, intermediários, $22,1 \%$, alta-dependência, 5,8\%, semi-intensivo, e 1,3\%, intensivo (Figura 1). Cabe mencionar que houve algumas variações do grau de dependência da admissão até a alta.

A recuperação do paciente e sua menor dependência da enfermagem dizem-nos que um bom prognóstico é aquele que leva o paciente ao autocuidado, à capacidade do ser humano em atender às suas necessidades básicas após a implementação do plano assistencial $^{(2)}$.

Portanto, o planejamento de alta deve ser visto como um processo onde as necessidades dos pacientes são identificadas e avaliadas e a assistência programada para preparar o paciente e/ou família para mover-se de uma posição passiva e receptora para uma posição ativa e de auto-responsabilidade ${ }^{(15)}$.

Analisando o grau de dependência do paciente conforme sua classificação realizada pelos enfermeiros estudados

Um dos nossos objetivos foi identificar, na evolução de enfermagem, o grau de dependência do paciente anotado pelo enfermeiro. Para tanto, encontramos, nas anotações dos enfermeiros, as classificações do grau de dependência do paciente como: independente, parcialmente dependente e totalmente dependente.

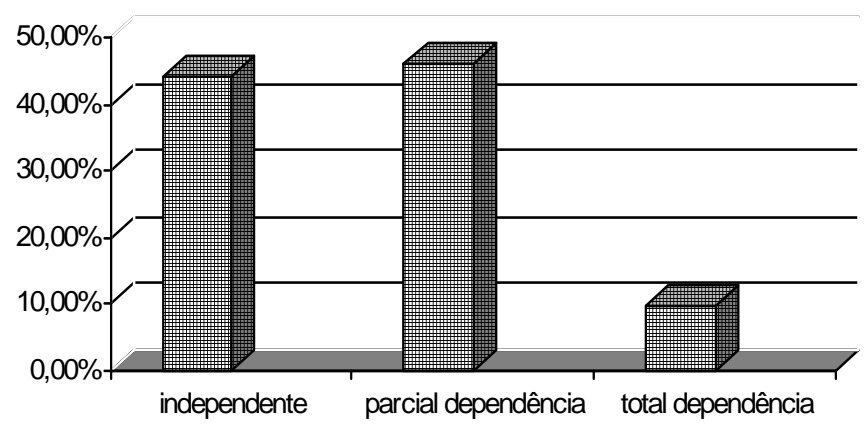

Figura 2 - Classificação dos pacientes internados nas Alas B1, C, D1A e D1B do Hospital de Clínicas de Marília - Unidade I, durante o período de 22 de setembro a 27 de outubro de 2000 , realizada pelos enfermeiros

Os mesmos pacientes foram também classificados pelos enfermeiros por meio de três níveis: independentes (44,1\%), parcialmente dependentes (46\%) e totalmente dependentes $(9,9 \%)$. Esses também apresentaram variações do grau de dependência, porém em menor porcentagem que a classificação realizada com o instrumento de classificação ${ }^{(4)}$.

As variações do grau de dependência ocorreram, principalmente, devido à instabilidade do quadro clínico do paciente.

Por meio dos dados obtidos, levantamos algumas hipóteses que tentavam questionar a relação entre o grau de dependência e as características apresentadas, tais como: idade, sexo, número de diagnóstico/paciente e tempo de permanência. Testando essas hipóteses, verificamos que os dados variaram muito de paciente para paciente, e não pudemos confirmar nenhuma delas. Ou seja, pacientes idosos possuíam variados graus de dependência, sendo que o número de diagnóstico também variou nas diversas faixas etárias. O grau de dependência também não aumentou conforme o número de diagnósticos.

Com relação ao tempo de permanência hospitalar, não pudemos comprovar que este aumenta conforme o grau de dependência, número de diagnóstico ou idade, mesmo porque a rotatividade de pacientes é grande, fazendo com que, assim que possível, o paciente receba alta e se recupere em seu domicílio.

A respeito do grau de dependência do paciente, não pudemos comparar os resultados dos achados nas anotações dos enfermeiros e a classificação, conforme o instrumento de classificação ${ }^{(4)}$, devido à não uniformidade de padrões entre os dois resultados, mesmo porque a classificação dos enfermeiros continha três níveis de grau de dependência e o instrumento de classificação(4) apresentava cinco níveis. Talvez, uma amostra maior de pacientes e uma uniformidade dos níveis de dependência corroborem algumas dessas hipóteses em estudos futuros.

Respostas dos enfermeiros

Pudemos verificar como os enfermeiros classificam os pacientes em grau de dependência dos cuidados de enfermagem por meio de questionário proposto.

Dos quinze respondentes, 14 eram do sexo feminino, e um, do sexo masculino, sendo 11 enfermeiros assistenciais e quatro docente-assistenciais. Constatamos que $80 \%$ desses profissionais estão na instituição há mais de 5 anos, e $66,7 \%$ se formaram há mais de 7 anos.

Observamos que $38,1 \%$ dos enfermeiros referiram 
que aprenderam a avaliar o grau de dependência do paciente na faculdade, e 38,1\% tiveram o aprendizado no dia-a-dia de sua prática assistencial. É importante mencionar que alguns enfermeiros buscaram esse conhecimento por meio de leituras complementares $(9,5 \%)$, ou leitura da Teoria de Wanda A. Horta $(9,5 \%)$ e por cursos a esse respeito $(4,8 \%)$.

Isso nos faz refletir sobre a formação do enfermeiro, principalmente com relação aos currículos ora vigentes nas Escolas de Enfermagem.

Os enfermeiros referiram que utilizam vários parâmetros para avaliar o grau de dependência, tais como: necessidade que o paciente apresenta para auxílio na deambulação, alimentação, higiene, movimentação, eliminações, vestir-se, condições de se autocuidar, diagnóstico médico, nível de consciência, presença do acompanhante, entre outros.

Não observamos, nas respostas dos enfermeiros, a necessidade do paciente em relação à oxigenação, controle de sinais vitais e terapêutica empregada, conforme sugere o instrumento de classificação ${ }^{(4)}$. Isso nos indica que essas três necessidades assistenciais (oxigenação, controle de sinais vitais e terapêutica indicada) não são avaliadas ou ainda estão latentes em seus julgamentos durante a classificação do paciente quanto ao grau de dependência.

Os indicadores de nível de consciência, estado mental e oxigenação são os principais fatores de peso na classificação, pois o nível de consciência e estado mental indicam a gravidade global do paciente ${ }^{(10)}$.

Em consoante com os dados apresentados, ainda podemos observar barreiras que dificultam a utilização de um sistema de classificação de pacientes, como a falta de clareza nesse processo ${ }^{(16)}$.

Nas unidades estudadas, pudemos observar que ainda há uma inadequação do processo de trabalho da enfermagem, principalmente relacionada à sistematização da assistência de enfermagem (SAE), o que implica uma precária avaliação do paciente e, conseqüentemente, do seu grau de dependência.

É necessário que os enfermeiros do estudo sejam

\section{REFERÊNCIAS BIBLIOGRÁFICAS}

1. Connor RJ. Effetive use of nursing resources: a research report. Hospitals 1961; 35(9):30-3.

2. Horta WA. O processo de enfermagem. São Paulo (SP): EPU/EDUSP; 1979. treinados para classificar o paciente e aplicados testes de confiabilidade para evitar contradições.

\section{CONCLUSÕES}

$\mathrm{Na}$ aplicação do instrumento de classificação(4), a maior incidência da classificação do paciente foi do nível intermediário, havendo discretas alterações do nível de dependência da admissão até a alta hospitalar.

Os enfermeiros classificaram os pacientes em três níveis: independente, parcial dependência e total dependência, sendo a maior incidência de pacientes independentes e parcialmente dependentes.

Não pudemos comparar os dados de classificação devido à não uniformidade de padrões entre os dados encontrados.

Os enfermeiros das unidades estudadas aprenderam a avaliar o grau de dependência na faculdade, no dia-a-dia da prática hospitalar, por meio de leituras complementares, na teoria de Wanda A. Horta e em cursos. Eles avaliam o grau de dependência observando a necessidade do paciente para deambulação, alimentação, higiene, movimentação, eliminações, condições de se autocuidar, diagnóstico médico, entre outros.

Conseguimos, com este trabalho, conhecer a realidade do grau de dependência do paciente e sua dimensão no processo assistencial da enfermagem do Hospital de Clínicas de Marília.

É necessário, sim, que o paciente seja classificado em seu grau de dependência, colaborando para um excelente planejamento da assistência de enfermagem, dimensionamento de recursos humanos e materiais, planejamento da assistência de enfermagem, previsão dos custos da assistência e melhor distribuição de atividades entre os membros da equipe de enfermagem.

Porém, para que isso ocorra, devemos continuar buscando uma forma simples, prática, rápida e eficiente para a classificação do grau de dependência do paciente, por meio da qualificação dos enfermeiros, buscando um instrumento próprio de avaliação.

3. Ribeiro CM. Sistema de classificação de pacientes para provimento de pessoal de enfermagem. [tese]. São Paulo (SP): Escola de Enfermagem/USP; 1972.

4. Fugulin FMT, Silva SH, Shimizu, HE, Campos FPF. Implantação do sistema de classificação de pacientes na unidade de Clínica Médica do Hospital Universitário de São Paulo. Rev Med HU-USP 1994; 4(1/2):63-8. 
5. Conselho Federal de Enfermagem (RJ). Resolução $N^{\circ}$ 189/96. Dispõe sobre os parâmetros para dimensionamento do quadro de profissionais de enfermagem nas instituições de saúde. Rio de Janeiro (RJ): Conselho Federal de Enfermagem; 1996.

6. Giovannetti P, Johnson JM. A new generation Patient Classification System. J Nurs Adm 1990 May; 20(5):33-40.

7. Gaidzinski RR. Dimensionamento de pessoal de enfermagem. In: Kurcgant P. Administração em enfermagem. São Paulo (SP): EPU; 1991. p. 91-6.

8. Fugulin FMT. Sistema de classificação de pacientes: análise das horas de assistência de enfermagem. [dissertação]. São Paulo (SP): Escola de Enfermagem/USP; 1997.

9. Martins EAP, Haddad MCL. Validação de um instrumento que classifica os pacientes em quatro graus de dependência do cuidado de enfermagem. Rev Latino-am Enfermagem 2000 abril; 8(2):74-82.

10. Perroca MG. Instrumento de classificação de pacientes de PERROCA: validação clínica. [tese]. São Paulo (SP): Escola de Enfermagem/USP; 2000.

11. Dal Bem LW. Instrumento para dimensionar horas diárias de assistência de enfermagem residencial. [dissertação]. São Paulo (SP): Escola de Enfermagem/USP; 2000.

12. Carmona LMP, Évora YDM. Sistema de classificação de pacientes: aplicação de um instrumento validado. Rev Esc Enfermagem USP. no prelo 2002.

13. Conselho Regional de Enfermagem de São Paulo (SP). Decisão COREN-SP/DIR/008/99: Normatiza a Implementação da Sistematização de Enfermagem - SAE nas Instituições de Saúde, no âmbito de Estado de São Paulo. São Paulo (SP): Conselho Regional de Enfermagem de São Paulo; 2000.

14. Conselho Nacional de Saúde (BR). Comissão Nacional de Ética em Pesquisa - CONEP. Resolução № 196/96. Dispõe sobre Diretrizes e Normas Regulamentadoras de Pesquisas envolvendo Seres Humanos. Governo Federal - Brasília (DF): Conselho Nacional de Saúde; 1996.

15. Marin MJS. Preparando o idoso para alta hospitalar. [tese]. Ribeirão Preto (SP): Escola de Enfermagem de Ribeirão Preto/ USP; 1999.

16. Rodrigues J Filho. Sistema de Classificação de Pacientes - Parte I: dimensionamento de pessoal de enfermagem. Rev Esc Enfermagem USP 1992 dezembro; 26(3):395-404. 